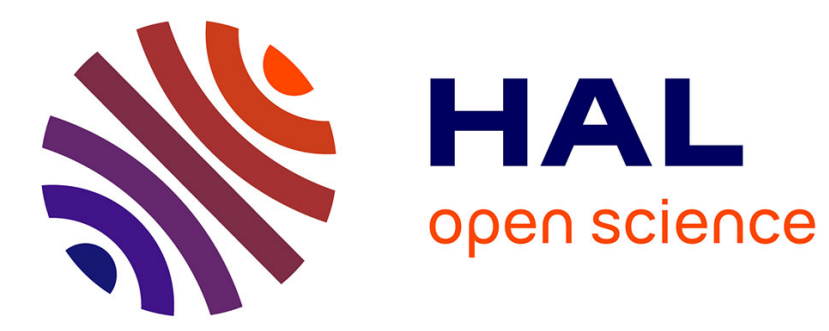

\title{
Une lampe flash coaxiale de faible inductance
}

F. Tfibel

\section{To cite this version:}

F. Tfibel. Une lampe flash coaxiale de faible inductance. Revue de Physique Appliquée, 1975, 10 (5), pp.331-333. 10.1051/rphysap:01975001005033100 . jpa-00243921

\section{HAL Id: jpa-00243921 https://hal.science/jpa-00243921}

Submitted on 1 Jan 1975

HAL is a multi-disciplinary open access archive for the deposit and dissemination of scientific research documents, whether they are published or not. The documents may come from teaching and research institutions in France or abroad, or from public or private research centers.
L'archive ouverte pluridisciplinaire HAL, est destinée au dépôt et à la diffusion de documents scientifiques de niveau recherche, publiés ou non, émanant des établissements d'enseignement et de recherche français ou étrangers, des laboratoires publics ou privés. 


\author{
Classification \\ Physics Abstracts \\ $0.640-6.700-0.650$
}

\title{
UNE LAMPE FLASH COAXIALE DE FAIBLE INDUCTANCE
}

\author{
F. TFIBEL
} Laboratoire de Photophysique Moléculaire du C. N. R. S.,
Université Paris-Sud, 91405 Orsay, France

(Reçu le 4 février 1975, révisé le 17 avril 1975, accepté le 21 avril 1975)

\begin{abstract}
Résumé. - On décrit une lampe flash tubulaire à double paroi dont le circuit de décharge est équivalent à un câble coaxial court-circuité à une extrémité. Reliée à un condensateur de faible self, la lampe (d'une longueur de $3 \mathrm{~cm}$ ) donne des flashes d'une durée à mi-hauteur de 1 à $2 \mu \mathrm{s}$ pour une énergie déchargée de $180 \mathrm{~J}$. Cette lampe a été utilisée comme source d'excitation dans des études de luminescence et également pour le pompage de lasers à colorants.
\end{abstract}

Abstract. - This note describes a double-walled tubular flash lamp characterized by a discharge path which is equivalent to the current path in a coaxial cable short-circuited at one end. The lamp (length $3 \mathrm{~cm}$ ) withstands discharges up to $180 \mathrm{~J}$ stored in a low-inductance capacitor. Flash duration at half-maximum is $1.5 \mu \mathrm{s}$ at maximum energy. The lamp has been used as an excitation source in luminescence studies and as an optical pump for dye lasers.

1. Introduction. - Dans de nombreuses applications des lampes flash (pompage optique de lasers, photolyse-flash, etc.), on cherche à obtenir des flashes de forte intensité et de courte durée. L'un des paramètres déterminant la durée du flash est la self-inductance du circuit de décharge, qui doit être aussi faible que possible. Claesson et Lindqvist [1] ont décrit une lampe flash annulaire dont la configuration coaxiale permettait d'obtenir une self-inductance très réduite (le principe en a été utilisé par la suite dans les premiers lasers à colorants pompés par flash [2]). Dans ce type de lampe, l'irradiation est effectuée au centre de la lampe annulaire. Toutefois, dans certains cas, cette disposition n'est pas réalisable. Nous décrivons dans la présente communication un type de lampe dont la selfinductance est également très faible mais dont la géométrie est semblable à celle des lampes linéaires couramment utilisées. Dans cette lampe, le circuit de décharge est équivalent à un câble coaxial courtcircuité à une extrémité, le conducteur étant constitué par le plasma. Ce principe a également été utilisé dans une réalisation décrite par Valloton et Wild [3].

2. Description. - La figure 1 montre une coupe du tube à décharge. Le courant de décharge est conduit par le plasma à l'intérieur du tube de quartz $\mathrm{B}(8,8 \mathrm{~mm}$ $11 \mathrm{~mm}$ ) et retourne par le plasma contenu entre les

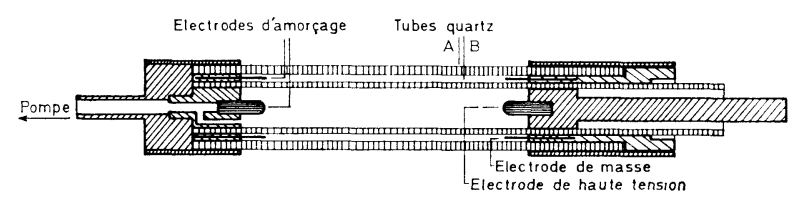

Fig. 1. - Coupe de la lampe-flash. A : tube de quartz extérieur ; $B$ : tube de quartz intérieur.

tubes de quartz B et A $(14,1 \mathrm{~mm}-18 \mathrm{~mm})$. La lampe est pourvue de quatre électrodes de tungstène : une axiale et une annulaire à chaque extrémité. Les deux électrodes situées à l'une des extrémités sont reliées entre elles par l'intermédiaire d'un support métallique commun (électrode d'amorçage). Les supports métalliques sont en alliage Invar. Les assemblages métalquartz sont effectués par collage à l'Araldite. Le tube de quartz intérieur (B) est rempli de gaz à travers un canal ménagé dans le support de l'électrode d'amorçage ; le gaz introduit pénètre à l'intérieur du second tube de quartz (A) grâce au jeu existant entre le tube de quartz intérieur et son logement dans ce même support. La distance entre les électrodes est de $3 \mathrm{~cm}$ (parcours total de la décharge : $6 \mathrm{~cm}$ ). La section du tube de quartz $\mathrm{B}$ est choisie égale à celle de l'espace annulaire compris entre les tubes de quartz $\mathbf{A}$ et $\mathbf{B}$ afin d'obtenir la même résistance dans les deux moitiés de la lampe. L'électrode d'amorçage est maintenue à un potentiel 
égal à la moitié de la tension de charge par un diviseur de tension résistif. L'amorçage est effectué en envoyant une impulsion haute tension sur cette électrode. Le tube est utilisé avec un remplissage d'oxygène ou d'un mélange oxygène-argon sous une pression de quelques cm Hg.

3. Caractéristiques électriques. - Le tube à décharge est relié directement au condensateur de décharge (Hivotronic, self-inductance $<5 \mathrm{nH}$ ) par une ligne plate de plaques de cuivre mince avec des feuilles de Mylar intercalées servant d'isolant. La mesure de la self-inductance de ce montage (condensateur et connexions) a donné $30 \mathrm{nH}$. Nous avons déterminé la self-inductance de la lampe elle-même ; celle-ci est de $25 \mathrm{nH}$, donc du même ordre de grandeur que la self du reste du circuit de décharge. Avec une self totale de $55 \mathrm{nH}$, le régime d'amortissement critique de la décharge est atteint pour une capacité de $2 \mu \mathrm{F}$. Ce type de lampe supporte des décharges électriques jusqu'à $180 \mathrm{~J}$.

4. Caractéristiques des éclairs. - 4.1 ProfiL D'INTENSITÉ DE L'ÉCLAIR. - Le profil de l'éclair est mesuré en envoyant la lumière diffusée de l'éclair à travers un monochromateur sur une photodiode rapide (CSF CPA 1143) dont le signal est visualisé sur un oscilloscope; la constante de temps du circuit de détection utilisé est inférieure à $0,1 \mu \mathrm{s}$. La figure 2

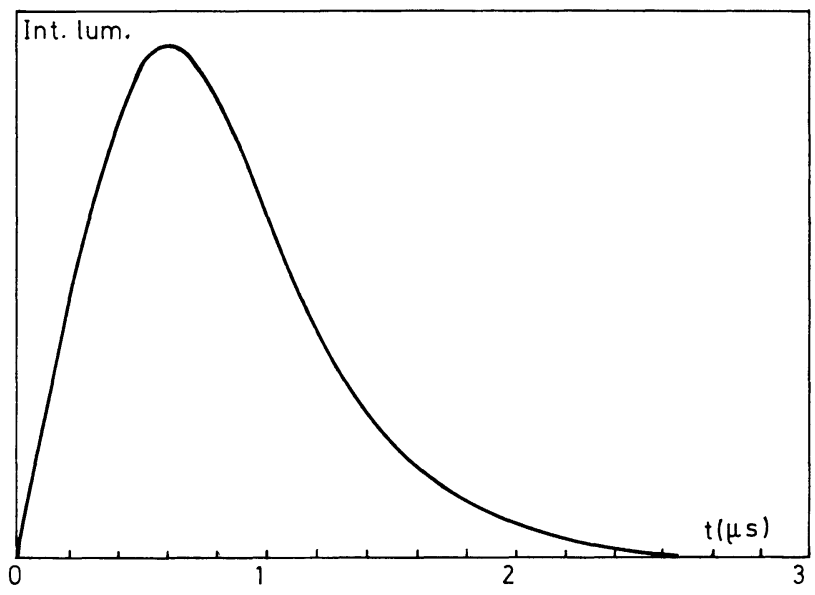

FIG. 2. - Profil d'intensité lumineuse d'un éclair de $64 \mathrm{~J}$ $(C=2 \mu \mathrm{F})$ dans un mélange oxygène-argon $(3: 1)$ sous 60 torr.

montre le profil d'un éclair de $64 \mathrm{~J}(\mathrm{C}=2 \mu \mathrm{F})$ dans un mélange oxygène-argon $(3: 1)$ sous 60 torr. Le temps de montée jusqu'à $90 \%$ du maximum d'intensité est de $0,4 \mu$ s. Les durées d'éclair à $1 / \mathrm{e}\left(\tau_{\mathrm{e}}\right)$ et $1 / 10\left(\tau_{10}\right)$ du maximum sont respectivement 1,1 et $1,8 \mu \mathrm{s}$. Jusqu'à une énergie déchargée de $100 \mathrm{~J}, \tau_{\mathrm{e}}$ reste pratiquement constante. $\tau_{10}$ (qui traduit l'importance de la traînée de l'éclair) augmente avec l'énergie électrique déchargée au-delà d'un seuil d'énergie électrique de $80 \mathrm{~J}$. Le choix de l'oxygène ou d'un mélange oxygène-argon à teneur élevée en oxygène comme gaz de remplissage a permis d'obtenir une traînée d'éclair très réduite [4].

4.2 ÉNERGIE LUMINEUSE. - La mesure de l'énergie lumineuse a été effectuée par actinométrie au ferrioxalate de potassium [5] dans les conditions d'utilisation de la lampe comme source d'excitation de fluorescence retardée : la cellule cylindrique contenant l'actinomètre $\left(l=30 \mathrm{~mm}, \varnothing_{\mathrm{i}}=10 \mathrm{~mm}\right)$ est placée parallèlement à la lampe, à une distance d'axe à axe de $45 \mathrm{~mm}$, à l'intérieur d'un cylindre isolant revêtu d'une couche diffusante de magnésie, de diamètre intérieur $\varnothing_{i}=118 \mathrm{~mm}$ et centré sur l'axe de la cellule. Le tableau I donne les valeurs du nombre $E$ de quanta absorbés entre 200 et $450 \mathrm{~nm}$ par les $2,4 \mathrm{~cm}^{3}$ d'actinomètre dans la cellule.

Pour une pression donnée du gaz de remplissage, l'énergie lumineuse de l'éclair est approximativement proportionnelle à l'énergie électrique déchargée.

Les rendements lumineux de l'air, l'oxygène et l'argon ont des valeurs voisines à pression et énergie déchargée identiques (pour des énergies $\leqslant 100 \mathrm{~J}$ ).

A titre de comparaison avec d'autres dispositifs d'excitation par éclairs, le taux de conversion à l'état triplet obtenu en excitant une solution d'anthracène par la lampe décrite est très voisin, pour une même énergie électrique déchargée, de celui obtenu en utilisant une source d'éclairs de type différent, de forte luminosité, mise au point par Lindqvist [6].

\section{Tableau I}

\section{Caractéristiques lumineuses des éclairs}

$\begin{array}{cccc}\text { Gaz } & \begin{array}{c}\text { Pression } \\ \text { (torr) }\end{array} & \begin{array}{c}\text { Energie } \\ (\mathrm{J})\end{array} & E \text { (quanta) } \\ \mathrm{O}_{2} \text { pur } & \overline{51} & \overline{64} & 1,5 \times 10^{17} \\ & 66 & 100 & 2,4 \times 10^{17} \\ \mathrm{O}_{2} 50 \% \text {-Ar 50\% } & 70 & 49 & 1,3 \times 10^{17} \\ & 70 & 64 & 1,8 \times 10^{17} \\ & 70 & 100 & 2,8 \times 10^{17}\end{array}$

5. Utilisation. - La caractéristique essentielle de ce type de lampe est de dissiper des énergies électriques élevées sur une faible longueur (jusqu'à $60 \mathrm{~J} / \mathrm{cm}$ sur $3 \mathrm{~cm}$ ) et en un temps très réduit (environ $1 \mu \mathrm{s}$ ). La limitation des dimensions de la lampe répond aux exigences rencontrées dans certaines études où l'échantillon étudié a une extension réduite, par exemple dans les études de luminescence. En assemblant plusieurs de ces lampes, déclenchées simultanément, autour de l'échantillon à irradier, on peut alors obtenir des flux d'excitation très élevés. Nous avons ainsi réalisé une source excitatrice constituée de 6 lampes identiques à celle décrite pour l'étude de la fluorescence retardée en phase liquide [7]. Cette lampe a également été utilisée pour le pompage optique de lasers à colorants [8]. 


\section{Bibliographie}

[1] Claesson, S. et Lindquist, L., Ark. Kemi 12 (1957) 1.

[2] Sorokin, P. P. et Lankard, J. R., IBM J. Res. Dev. 11 (1967) 148.

[3] Vallotton, M. et Wild, U. P., J. Phys. E 4 (1971) 417.

[4] Claesson, S. et LindQvist, L., Ark. Kemi 11 (1957) 535.
[5] PARKer, C. A., Photoluminescence of solutions (Elsevier, Amsterdam, 1968) 208.

[6] Lindqvist, L., Revue Phys. Appl. 3 (1968) 15.

[7] Tfibel, F. et LindQvist, L., Chem. Phys., à paraître.

[8] SoEP, B., Opt. Commun. 1 (1970) 433. 